\title{
Rolling away from the Wall into Granular Matter
}

\author{
V. L. Díaz-Melián, A. Serrano-Muñoz®, M. Espinosa, L. Alonso-Llanes, G. Viera-López®, and E. Altshuler®* \\ Group of Complex Systems and Statistical Physics, Physics Faculty, University of Havana, 10400 Havana, Cuba
}

(Received 23 December 2019; revised 14 March 2020; accepted 16 July 2020; published 12 August 2020)

\begin{abstract}
The sedimentation of solid objects into granular matter near boundaries is an almost virgin field of research. Here we describe in detail the penetration dynamics of a cylindrical object into a quasi-2D granular medium. By tracking the trajectory of the cylinder as it penetrates the granular bed, we characterize two distinct kinds of motion: its center of mass moves horizontally away from the lateral wall, and it rotates around its symmetry axis. While the repulsion is caused by the loading of force chains between the intruder and the wall, the rotation can be associated to the frictional forces between the grains and the intruder. Finally, we show the analogies between the sedimentation of twin intruders released far from any boundaries, and that of one intruder released near a vertical wall.
\end{abstract}

DOI: 10.1103/PhysRevLett.125.078002

Fluid and solidlike behaviors tend to coexist in the granular state of matter. For example, the penetration of a solid intruder released into a wide granular bed may be quite smooth for the first part of its trajectory, but eventually becomes fluctuating, until the object completely stops at a certain depth inside the material. The first part of the motion may resemble the penetration of a sphere into a viscous fluid, but then the medium experiences a transition to the jammed state [1-5], which distinguishes the process from any expectable behavior of a liquid. Real life scenarios such as balls mills [6] and excavations near walls [7] involve complex phenomena whose handling would benefit from a better understanding of how intruders penetrate near boundaries in granular matter.

However, in spite of the substantial research efforts devoted to understand the impact and penetration of lowvelocity projectiles into granular beds [8-19], intruder-wall interactions have rarely been documented in the literature. A few authors have studied the effect of confinement in cylindrical containers where the intruder penetrates at the center. That is the case of Seguin et al. in 2008, who concludes that the final penetration depth of a sphere increases with the radius of the container, and that the effects of the walls decay within a distance of the order of the sphere's diameter [20]. In 2010, von Kann et al. studied the effect of the container radius on the shape and strength of sand jets occurring in loosely packed sand [21]. Katsuragi reported the quasistatic penetration of a spherical intruder into a cylindrical granular column, and found scaling laws relating the drag force and the wall pressures [22]. To our knowledge, the effect of a single vertical wall on the motion of an intruder has been studied only once: In 2008, Nelson et al. reported the horizontal repulsion of the intruder away from the wall by recording its landing position [11].

Here, we perform systematic penetration experiments of a cylindrical intruder into a quasi-2D granular medium, which reveal its detailed motion after being released at the free granular surface near a vertical wall. We observe two distinct phenomena during the sedimentation process: the intruder separates from the wall in the horizontal direction, and rotates around its symmetry axis. Further experiments suggest that the repulsion and rotation of one intruder released near a vertical wall is equivalent to substituting the wall by a second intruder released side by side with the original one.

Millimetric-sized expanded polystyrene spheres where deposited into a Hele-Shaw cell, as shown in Fig. 1(a). Cylindrical intruders were released from the surface of the granular bed, starting at different horizontal distances from the left (or right) wall of the cell by means of an electromagnetic device that minimized spurious vibrations and torques on the intruder when released. The penetration process was followed using a digital camera that took videos through one of the large faces of the cell. Two colored dots situated at the center and near the border of one circular face of the cylinder [Fig. 1(b)] served as reference points for image analysis. Using them, the motion of the intruder's center of mass and its angle of rotation could be measured. Supplemental Material [23] provided with this work explains in depth the experimental details. Some sample videos of three different experimental configuration are included.

Figure 1(c) shows the trajectories of the intruder after being released at different initial distances from the wall, $x_{0}$ (notice that the distance from the vertical wall has been plotted as the position of the left edge of the intruder relative to the boundary). Our results not only confirm that the intruder is laterally repelled by the wall (as suggested in Ref. [11], based on the final position of the object), but also reveal the details of the whole penetration process. For all values of $x_{0}$, the first stage of the penetration consists of an almost purely vertical plunge, after which the lateral motion 


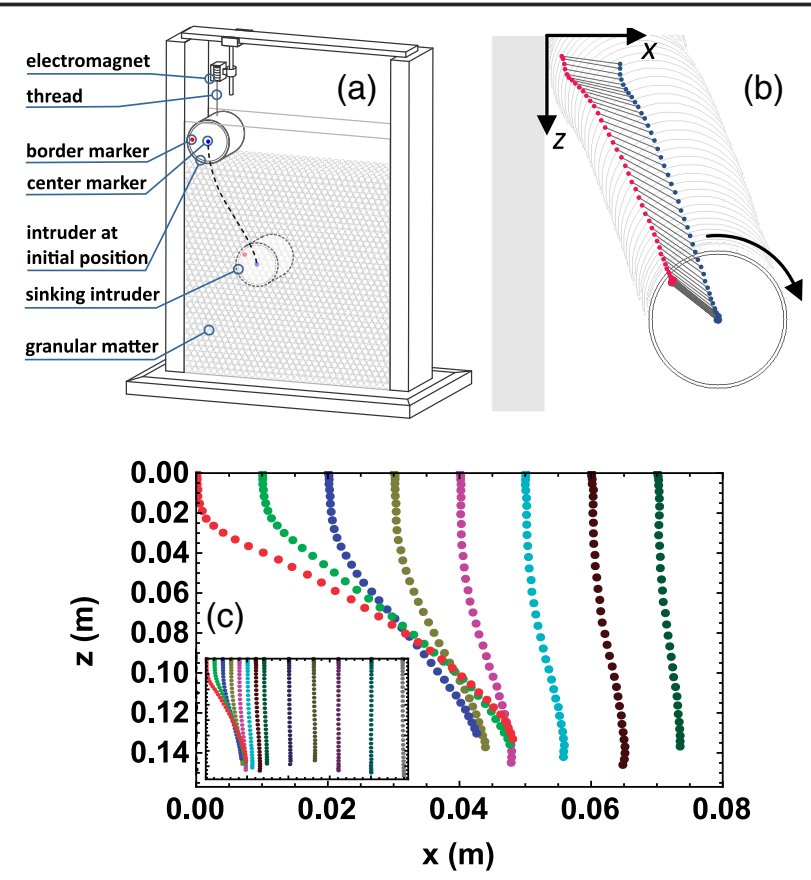

FIG. 1. Rolling away from the wall. (a) Cylinders are released into a Hele-Shaw cell at different initial distances $x_{0}$ from the left wall (the cylinder path is sketched for $x_{0}=0$ ). (b) Tracking of center (blue) and border (red) markers. The curved arrow indicates the rotation of the intruder in the clockwise direction. (c) From left to right: trajectories of an intruder released at $x_{0}=0,1,2,3,4,5,6$, and $7 \mathrm{~cm}$, respectively (the inset includes $x_{0}=10,13,16,20$, and $23.75 \mathrm{~cm}$ ). Intruder mass and diameter were $0.255 \mathrm{~kg}$ and $7.5 \mathrm{~cm}$, respectively. All trajectories were averaged over 10 measurements.

away from the wall takes place. When the intruder is released relatively near the wall, the repulsion is smaller as the initial position of the cylinder, $x_{0}$ is larger. However, for even larger values of $x_{0}$ the sinking can be almost vertical, so the effect of the wall is no longer felt. Figure 1(c) also suggests that the final penetration depth does not depend substantially on $x_{0}$.

Figure 2(a) reports the time evolution of the net horizontal position of the intruder, confirming that it increases as $x_{0}$ decreases. The inset shows another interesting feature of the penetration process: the maximum lateral displacement from the wall is almost constant for small values of $x_{0}$, and then grows linearly starting approximately at $x_{0}=5 \mathrm{~cm}$. That behavior suggests that the interaction between the wall and the intruder decays significantly when the intruder is away from the wall a distance approximately equal to $l=5 \mathrm{~cm}$. We will discuss the physical meaning of this value later on.

Figure 2(b) quantifies an entirely novel phenomenon: as the intruder moves away from the wall, it rotates around its symmetry axis. The graph shows that, if $x_{0}$ is small enough (i.e., 0 and $1 \mathrm{~cm}$ ), the rotation is relatively large and clockwise, while it is smaller and counterclockwise for $2 \mathrm{~cm}<x_{0} \leq 7 \mathrm{~cm}$. After $x_{0} \simeq 10 \mathrm{~cm}$, the rotation is
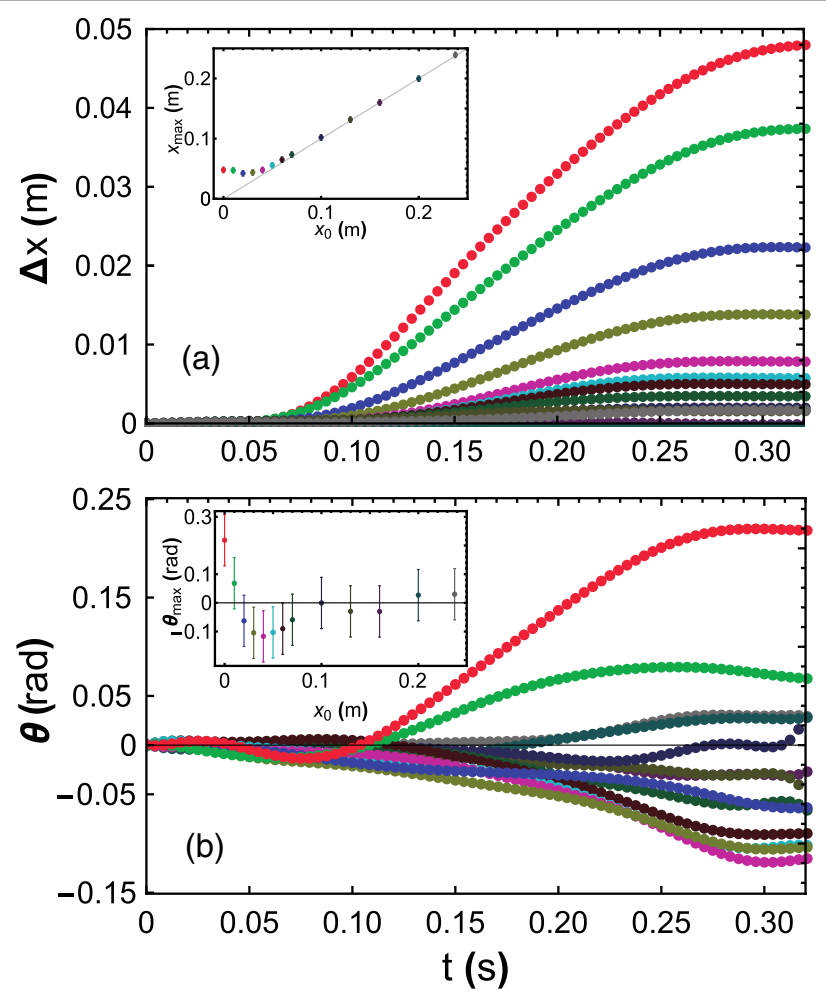

FIG. 2. Details of the horizontal and rotational motion seen in the experiments. (a) Time evolution of the lateral displacements $\left(\Delta x=x-x_{0}\right)$ of the intruder for the $x_{0}$ values shown in Fig. 1(c). In the inset, the maximum lateral separation from the wall as a function of $x_{0}$ is shown (the line follows $x_{\max }=x_{0}$ ). (b) Time evolution of the angle rotated by the intruder (relative to its initial value). In the inset, the maximum angle of rotation as a function of $x_{0}$ is shown. Each curve in (a) and (b) are averaged over 10 repetitions of each experiment. Points in the insets are also averaged over 10 repetitions (error bars are the corresponding standard deviations). The color scale of both (a) and (b) is consistent with the one shown in Fig. 1.

negligible, as clearly shown in the inset, strongly suggesting that the repulsion and rotation effects are correlated. In the rest of the document, and also in the Supplemental Material [23], we will avoid using the terms clockwise and counterclockwise, since they depend on the side of the cell from which the intruder is released. Instead, we will call "normal" the rotational motion expected if the intruder was rolling down a solid ramp with friction while moving away from the wall. So, our experiments show that the cylinder describes "normal rotation" for $x_{0}=0$ and $1 \mathrm{~cm}$, and "anomalous rotation" for $2 \mathrm{~cm}<x_{0} \leq 7 \mathrm{~cm}$.

Now, we discuss the physics behind the penetration process, with emphasis in repulsion and rotation phenomena. Figure 3(a) shows a snapshot from 2D discrete element simulations (DEM). It illustrates the presence of force chains underneath the intruder that are involved in the penetration dynamics. It is possible to see stronger force chains between the intruder and the wall than those at the opposite side of the intruder (simulation details can be 

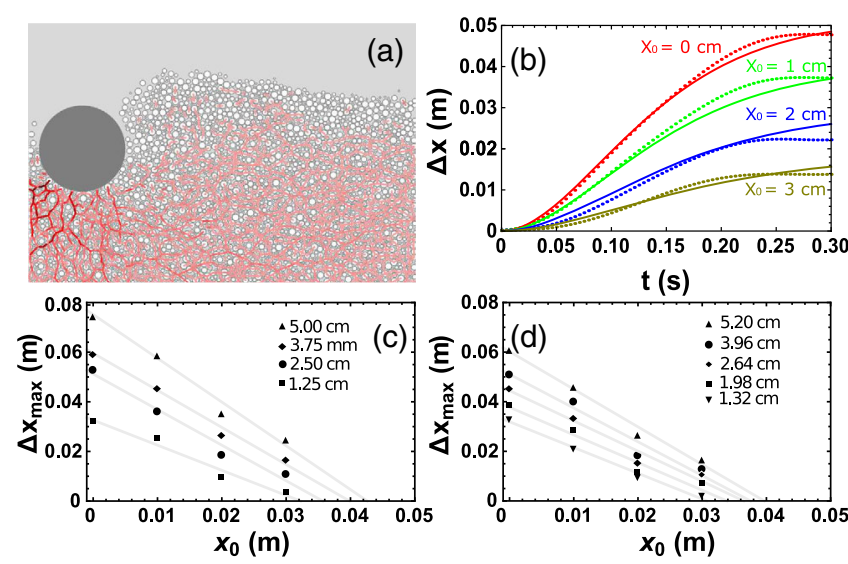

FIG. 3. DEM simulations and modeling of the repulsion. (a) Snapshot from a DEM simulation showing the force chains during the penetration of a disk-shaped intruder near a wall just after being released (darker red indicates bigger forces). (b) Horizontal motion vs time for $x_{0}=0$ : circles and lines represent experiment and the output of Eq. (1), respectively. (c) Maximum horizontal separation vs $x_{0}$ for different cylinder radii: symbols and lines represent DEM simulations and linear fits, respectively. (d) Maximum horizontal separation vs $x_{0}$ for different cell thicknesses: symbols represent DEM simulations.

found in Supplemental Material [23]). It qualitatively confirms the speculation by Nelson et al. [11] related to the displacement along the $x$ axis: "the motion of the projectile serves to load force chains between [intruder] and wall, which causes the repulsion."

Let us first try to understand the cause of the horizontal motion by analyzing our experimental data. Careful observation of the videos in slow motion shows that, immediately after the release of the intruder, partially jammed grains located between the cylinder and the vertical boundary "push" the intruder away from the wall. However, a short time afterwards, the grains are fully mobilized around the intruder, and the repulsive force substantially decays (see Supplemental Material [23] for further details). We will assume that the pushing forces acting on the intruder are exerted by force chains of finite length showing an effective elastic behavior. We condense this picture into a simple 2D mechanical model: the intruder is assumed as a disk of radius $R$ where linear springs with equilibrium length $l$ are radially attached to its lower half. We do not consider any torsional effects on the springs, so their only degree of freedom is associated to compression. If the springs are not touching any cell boundaries, they do not transmit any force from them to the intruder. So, if the intruder's left boundary is away from the vertical wall a distance $x>l$, it will not "feel" the presence of the wall, so the only horizontal force felt by the intruder will be a velocity-dependent drag. A similar analysis allows us to disregard the effect of the bottom wall. Assuming a continuum of finite springs for an intruder near the wall, and including a viscouslike force, we get the following equation of motion for the intruder's center of mass, $x_{c}$ (see details of the derivation in Supplemental Material [23]):

$m \ddot{x}_{\mathrm{c}}=k\left\{\left[(l+R)^{2}-x_{\mathrm{c}}^{2}\right]^{1 / 2}-x_{\mathrm{c}} \cos ^{-1} \frac{x_{\mathrm{c}}}{l+R}\right\}-f\left(\dot{x}_{c}\right)$,

where $R$ is the radius of the cylinder, $l=5 \mathrm{~cm}$ (see discussion above), and $f\left(\dot{x}_{c}\right)=\alpha \dot{x}_{c}\left(1+\beta \dot{x}_{c}\right)$, where $\alpha=$ $6 \pi \eta R$ and $\beta=(3 / 8)(\rho R / \eta) ; \rho$ is taken as the granular density times the packing fraction. The remaining constants $k$ and $\eta$ were freely changed in the model to reproduce the averaged experimental results for the values of $x_{0}=0,1,2$, $3 \mathrm{~cm}$. After fitting the data we obtained $k=35 \mathrm{~N} / \mathrm{m}$ and $\eta=5.0 \mathrm{~kg} \mathrm{~m}^{-1} \mathrm{~s}^{-1}$.

Notice that in Eq. (1), the first term at the right only holds for $R \leq x_{c} \leq l+R$. The continuous lines in Fig. 3(b) show the solutions of Eq. (1) for $x_{0}=0,1,2$, and $3 \mathrm{~cm}$ (note that $\left.\Delta x=x_{c}-R-x_{0}\right)$. As can be seen, the model reproduces quantitatively the experimental curves within root-meansquare errors of $0.0214,0.0483,0.0514$, and 0.0551 (normalized to the maximum value of each experimental curve) for $x_{0}=0,1,2$, and $3 \mathrm{~cm}$, respectively. The best accuracy is achieved for small values of $x_{0}$, where the repulsion is more evident. All in all, it can be said that the lack of symmetry of the force chains distribution around the intruder, their rapid decay, and a velocity-dependent "granular viscosity," are the key ingredients to explain the repulsion of the intruder by the wall.

Let us briefly discuss the physical meaning of our model parameters. First, we assume that the breaking and building of force chains during the penetration process can be characterized by an elastic constant $k$ which is much smaller than that expectable for grain-grain interactions, or even than that associated to linear chains of grains behaving elastically [30]. In fact, our "elastic interaction" cannot be taken literally, since the true repulsion mechanism is largely associated to irreversible plastic deformations of the granular system.

In our model, the springs have an average length $l$. We speculate that it corresponds to the horizontal size of the stress field associated to the intruder (HSFI) [31]. In order to connect $l$ with other lengths involved in our system, we have performed DEM simulations. The symbols in Fig. 3(c) correspond to simulated $\Delta x_{\max }$ vs $x_{0}$ graphs for different diameters of the intruder, where $\Delta x_{\max }$ is the value of $x-x_{0}$ when the intruder stops. Based on them, we can make a rough estimation of $l$ as the values of $x_{0}$ corresponding to $\Delta x_{\max }=0$. As indicated by the linear fits represented by straight lines, $l$ increases with the intruder's radius, which is consistent with the fact that the HSFI is reported to increase with the horizontal size of the intruder [31]. The symbols in Fig. 3(d) correspond to simulated $\Delta x_{\max }$ vs $x_{0}$ graphs for different thicknesses of the Hele-Shaw cell (or, 
equivalently, to different heights of the cylindrical intruder). As can be seen, the maximum interaction distance also increases with the cell width within the range of our simulations.

Finally, let us comment on the "viscosity factor" that multiplies the velocity squared in Eq. (1). Its value $\alpha \beta=$ $0.09 \mathrm{~kg} / \mathrm{m}$ fits well our experimental data, but it is approximately 2-3 times larger than the equivalent parameter used by Pacheco et al. [13] to reproduce the vertical penetration of a spherical intruder into a cylindrical silo filled up with the same kind of granular matter. This fact suggests that the granular viscosity term is not inertial in the hydrodynamic sense: the intruder collides with "grain clusters" defined by force chains [32,33], so it is indirectly influenced by the specific geometry-and dimensionality —of the experiment.

Now, we introduce some preliminary ideas to model the normal rotation. The comparison between Figs. 2(a) and 2(b) suggests that the normal rotation is caused by the horizontal motion. We also assume that nonzero frictional forces between the surface of the intruder and the surrounding grains are essential to get the rotational effect, which was corroborated by DEM simulations. As the intruder moves laterally with a velocity $\dot{x}(t)$, it experiences tangential frictional forces pointing to the wall that are stronger at the bottom than at the top, due to Janssen's effect (see Supplemental Material [23]). We will assume that those forces are proportional to $\left(1-e^{-z / \lambda}\right)$, where $\lambda$ is a characteristic length of the order of the width of the container [13] (5.3 cm in our case). Then, the difference between the bottom and top horizontal shear forces acting on an intruder whose center of mass is located at $(x, z)$ scales as $F_{\text {bottom }}-F_{\text {top }} \sim \mu_{\text {eff }} e^{-z / \lambda}$, where $\mu_{\text {eff }}$ is an effective friction that has been found proportional to the penetration velocity of an intruder into a fluidized granular bed [34], $\mu_{\text {eff }}=\xi \dot{x}$. Then, the equation describing the rotational motion of the cylinder can be written as $\ddot{\theta}=k_{r} \dot{x} \exp (-z / \lambda)-f_{\theta} \dot{\theta}$, where $k_{r}$ depends on the properties of the granular medium and the geometry of the experiment, and $f_{\theta}$ is proportional to the frictional torque exerted by the grains on the intruder. Using $k_{r}=$ $400 \mathrm{~m}^{-1} \mathrm{~s}^{-1}$ and $f_{\theta}=32 \mathrm{~s}^{-1}$ as free parameters, and inserting $x(t)$ taken from the experiments, the above equation of motion reproduces quite well the experimental $\theta$ vs $t$ dependence for $x_{0}=0$ (see Supplemental Material [23]). However, this approach to explain the rotation is not able to reproduce properly the experimental results for $x_{0} \geq 1 \mathrm{~cm}$, including the anomalous ones (interestingly, anomalous rotations cannot be reproduced by our DEM simulations, as stated in the Supplemental Material [23]). The anomaly may be caused by "quenched" effects, such as the inhomogeneous deposition of grains in the cell (especially near walls) associated with our filling protocol. But it can be also associated with "dynamical" effects, like the inversion of the effective tangential friction due to

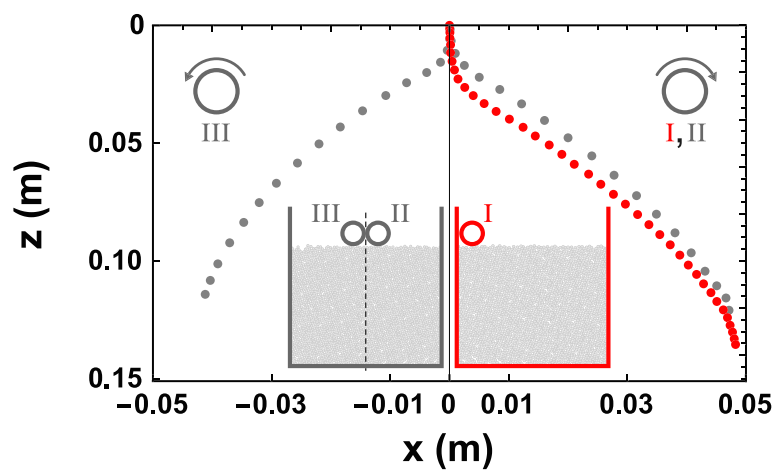

FIG. 4. Intruder-wall vs intruder-intruder interactions. The red circles correspond to the trajectory of a single intruder released near the left wall $\left(x_{0}=0 \mathrm{~cm}\right)$ while the gray ones correspond to the case of two intruders released at the center of the Hele-Shaw cell, far from the edges (see lower inset). The upper insets show the rotation direction of the intruders: clockwise for the trajectories at the right, and counterclockwise for the one at the left. The points are averages over 10 repetitions of each experiment.

nontrivial flows of grains around the intruder when it is released farther from the wall-where force chains are less relevant. Such study will be the subject of future research.

We present a final experiment showing the parallelism between the motion of a single intruder released near a vertical wall, and two identical intruders released side by side far from any walls. Two cylinders identical to the one that produced all previous data were released side by side at the center of the Hele-Shaw cell, i.e., far from the lateral walls. As a result, they repel each other, producing two symmetrical trajectories represented by gray dots in Fig. 4 (this fact has been reported by Pacheco-Vázquez and RuizSuárez in a purely 2D system [12], and earlier by Nelson et al. [11] by observing the end points of the two trajectories). In the same figure we have plotted a single intruder released at $x_{0}=0 \mathrm{~cm}$ from the left wall, represented by red circles. The very good coincidence between the red and right gray trajectories suggests that the evolution of force chains between the two intruders is quite similar to the ones felt by the intruder at the right if the one at the left is substituted by a flat wall. This nontrivial fact resembles the method of images for electrical charges [35] and flow fields near boundaries in hydrodynamics [36] with recent applications to the case of microbial swimmers [37,38].

In summary, we have fully characterized the horizontal repulsion of solid intruders when penetrating a quasi-2D granular bed near a wall, after being released on the free granular surface at a distance $x_{0}$ from a vertical boundary. Our experiments reveal that the interaction with the wall produces rotation of the intruder around its symmetry axis, which is normal or anomalous for small or large values of $x_{0}$, respectively. We are able to reproduce the motion along the $x$ axis by assuming that the force chains associated with 
jammed grains between the wall and the intruder "push" it away from the wall while it is stopped by granular viscouslike forces. We assume that the nonzero frictional forces between the surface of the intruder and the surrounding grains are essential to get the rotational effect, which was corroborated by DEM simulations. Finally, we suggest that the repulsion and rotation of one intruder released near a vertical wall can be largely reproduced by substituting the wall by a second intruder released side by side with the original one.

We acknowledge D. J. Durian calling our attention to intruder-wall interactions as well as useful discussion with E. Clément, A. García, and T. Shinbrot. This research was performed under the institutional project "Granular media: creating tools for the prevention of catastrophes."

*ealtshuler@fisica.uh.cu

[1] A. J. Liu and S. R. Nagel, Nature (London) 396, 21 (1998).

[2] A. S. Keys, A. R. Abate, S. C. Glotzer, and D. J. Durian, Nat. Phys. 3, 260 (2007).

[3] C. P. Goodrich, A. J. Liu, and J. P. Sethna, Proc. Natl. Acad. Sci. U.S.A. 113, 9745 (2016).

[4] Y. Jin, P. Urbani, F. Zamponi, and H. Yoshino, Sci. Adv. 4, eaat6387 (2018).

[5] Y. Zhao, J. Barés, H. Zheng, J. E. S. Socolar, and R. P. Behringer, Phys. Rev. Lett. 123, 158001 (2019).

[6] R. Rajamani, B. Mishra, R. Venugopal, and A. Datta, Powder Technol. 109, 105 (2000).

[7] C. Yoo, J. Geotech. Geoenviron. Eng. 127, 225 (2001).

[8] R. Mikkelsen, M. Versluis, E. Koene, G.-W. Bruggert, D. Van Der Meer, K. Van Der Weele, and D. Lohse, Phys. Fluids 14, S14 (2002).

[9] J. S. Uehara, M. A. Ambroso, R. P. Ojha, and D. J. Durian, Phys. Rev. Lett. 90, 194301 (2003).

[10] D. I. Goldman and P. Umbanhowar, Phys. Rev. E 77, 021308 (2008).

[11] E. L. Nelson, H. Katsuragi, P. Mayor, and D. J. Durian, Phys. Rev. Lett. 101, 068001 (2008).

[12] F. Pacheco-Vázquez and J. Ruiz-Suárez, Nat. Commun. 1, 123 (2010).

[13] F. Pacheco-Vázquez, G. A. Caballero-Robledo, J. M. Solano-Altamirano, E. Altshuler, A. J. Batista-Leyva, and J. C. Ruiz-Suárez, Phys. Rev. Lett. 106, 218001 (2011).

[14] J. Ruiz-Suárez, Rep. Prog. Phys. 76, 066601 (2013).

[15] T. A. Brzinski III, P. Mayor, and D. J. Durian, Phys. Rev. Lett. 111, 168002 (2013).

[16] E. Altshuler, H. Torres, A. González-Pita, G. SánchezColina, C. Pérez-Penichet, S. Waitukaitis, and R. Hidalgo, Geophys. Res. Lett. 41, 3032 (2014).
[17] G. Sánchez-Colina, L. Alonso-Llanes, E. Martínez, A. Batista-Leyva, C. Clement, C. Fliedner, R. Toussaint, and E. Altshuler, Rev. Sci. Instrum. 85, 126101 (2014).

[18] C. S. Bester and R. P. Behringer, Phys. Rev. E 95, 032906 (2017).

[19] H. Katsuragi and D. J. Durian, Nat. Phys. 3, 420 (2007).

[20] A. Seguin, Y. Bertho, and P. Gondret, Phys. Rev. E 78, 010301(R) (2008).

[21] S. von Kann, S. Joubaud, G. A. Caballero-Robledo, D. Lohse, and D. van der Meer, Phys. Rev. E 81, 041306 (2010).

[22] H. Katsuragi, Phys. Rev. E 85, 021301 (2012).

[23] See Supplemental Material at http://link.aps.org/ supplemental/10.1103/PhysRevLett.125.078002 for experimental details, derivation of Eq. (1), preliminary modeling of the rotational motion, and description of DEM simulations which includes Refs. [19,24-29].

[24] L. Landau and E. Lifshitz, in Theoretical Physics, Fluid Mechanics Vol. 6 (Pergamon, London, 1987).

[25] N. V. Brilliantov, F. Spahn, J.-M. Hertzsch, and T. Pöschel, Phys. Rev. E 53, 5382 (1996).

[26] L. E. Silbert, D. Ertaş, G. S. Grest, T. C. Halsey, D. Levine, and S. J. Plimpton, Phys. Rev. E 64, 051302 (2001).

[27] H. P. Zhang and H. A. Makse, Phys. Rev. E 72, 011301 (2005).

[28] S. Plimpton, Technical Report, Sandia National Labs., Albuquerque, NM, USA, 1993.

[29] Project Chrono, http://projectchrono.org, accessed: 201603-07.

[30] B. Andreotti, Y. Forterre, and O. Pouliquen, Granular Media: Between Fluid and Solid (Cambridge University Press, Cambridge, England, 2013).

[31] W. Kang, Y. Feng, C. Liu, and R. Blumenfeld, Nat. Commun. 9, 1101 (2018).

[32] Y. Takehara, S. Fujimoto, and Okumura, Europhys. Lett. 92, 44003 (2010).

[33] A. H. Clark, A. J. Petersen, and R. P. Behringer, Phys. Rev. E 89, 012201 (2014).

[34] R. Harich, T. Darnige, E. Kolb, and E. Clément, Europhys. Lett. 96, 54003 (2011).

[35] J. D. Jackson, Classical Electrodynamics (John Wiley \& Sons, Inc, New York, 1999).

[36] J. Blake, in Mathematical Proceedings of the Cambridge Philosophical Society (Cambridge University Press, Cambridge, England, 1971), Vol. 70, pp. 303-310.

[37] E. Lauga and T. R. Powers, Rep. Prog. Phys. 72, 096601 (2009).

[38] E. Altshuler, G. Miño, C. Pérez-Penichet, L. del Río, A. Lindner, A. Rousselet, and E. Clément, Soft Matter 9, 1864 (2013). 